\title{
High Gradient Cryomodule Prototype for the International Linear Collider
}

Daniel Bafia, IIT

FERMILAB-POSTER-20-002-TD

\section{Necessity of High Gradient/High $Q_{0}$ for ILC Realization}

- The world is ready for a new Higgs factory for BSM physics

- ILC is a ready to go technology:

- Uses SRF cavities; capable of very high $Q_{0}$ and gradients

- Largest cost driver is the average accelerating gradient of the main SRF LINAC

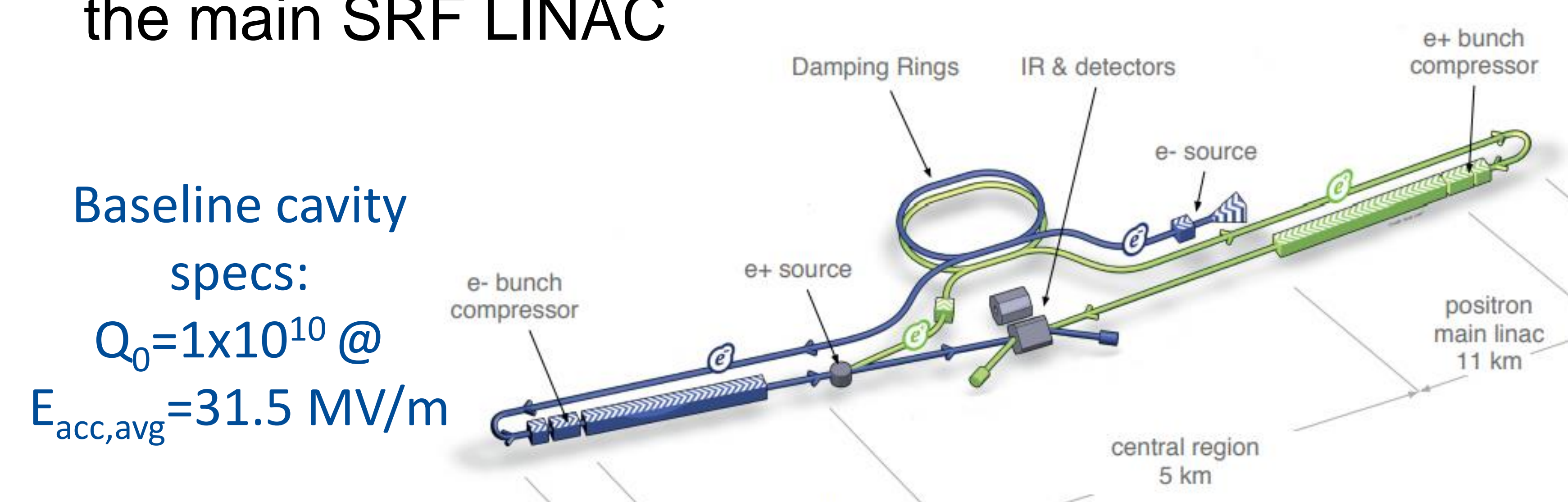

$\mathrm{E}_{\mathrm{acc}, \mathrm{avg}}=31.5 \mathrm{MV} / \mathrm{m}$

- Increasing the baseline cavity specs allows for either:

\section{Lower Cost}

Cost Estimation of 250GeV ILC LINAC

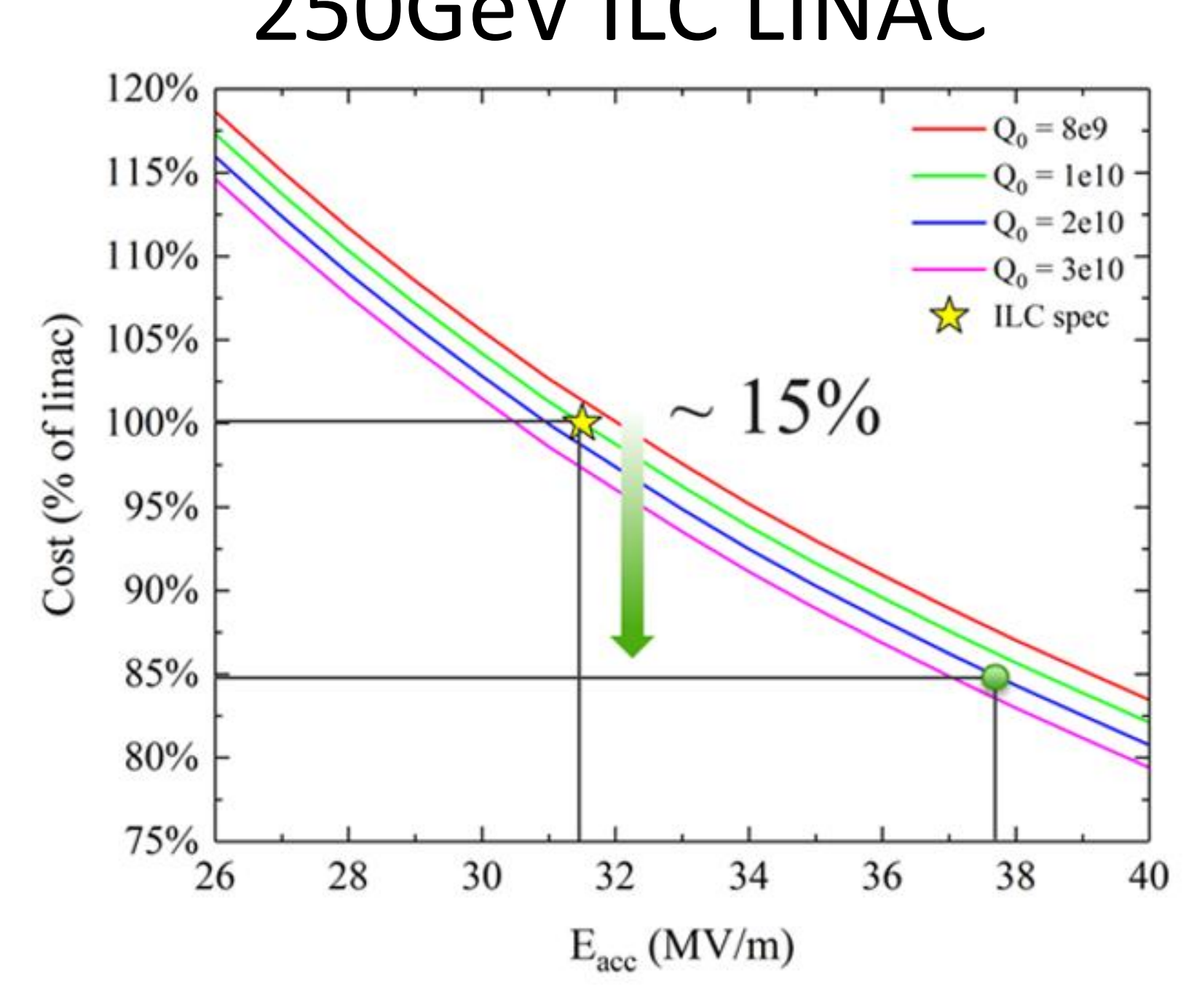

Increasing:

$\mathrm{E}_{\mathrm{acc}, \mathrm{avg}}=31.5 \rightarrow 38 \mathrm{MV} / \mathrm{m}$ $Q_{0}=1 \times 10^{10} \rightarrow 2 \times 10^{10}$ allows for a $15 \%$ reduction in cost of main SRF LINAC

\section{Higher Luminosity}

FNAL Workshop on HL-HG ILC:

- Proposed a high luminosity/high gradient ILC upgrade enabled by recent progress made in High $G / Q_{0} R \& D$ :

\begin{tabular}{|c|c|c|c|}
\hline & TDR Baseli & 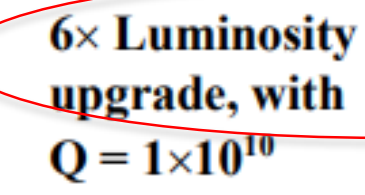 & 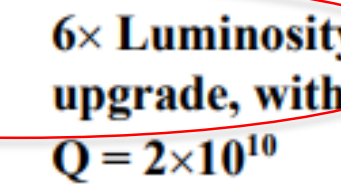 \\
\hline Energy $\mid$ GeVl] & 250 & 250 & 250 \\
\hline | Luminosity $\left|\times 10^{34}\right|$ & 1.35 & 8.1 & 8.1 \\
\hline 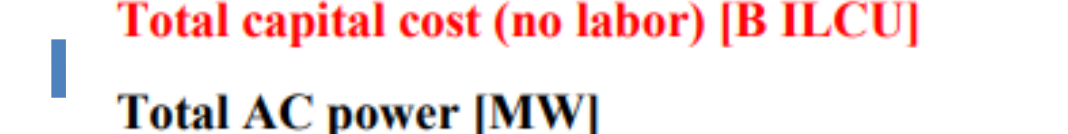 & 5.5 & 8.0 & 7.73 \\
\hline II Cyomodulus (including cavities) [B ILCV| & 1.93 & ${ }_{1.93}^{200}$ & ${ }_{1.93}^{207}$ \\
\hline 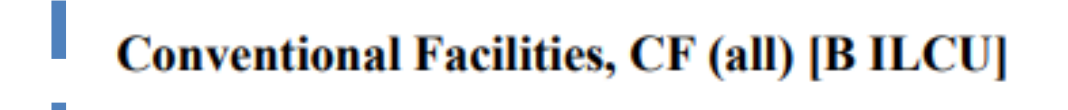 & 1.43 & 1.63 & 1.63 \\
\hline | Refrigeration system [B LLCU] & 0.5 & 1.3 & 1.0 \\
\hline | High power RF (linac only) [B LLCV] & 0.6 & 1.2 & 1.2 \\
\hline | Damping ring [ [ L LCVI] & & 0.66 & 0.66 \\
\hline 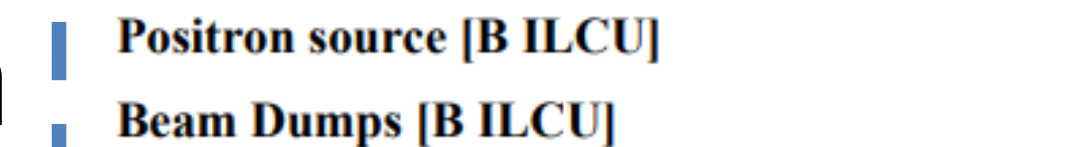 & $\begin{array}{l}0.23 \\
0.07 \\
-10\end{array}$ & 0.69 & $\begin{array}{l}0.69 \\
0.21\end{array}$ \\
\hline 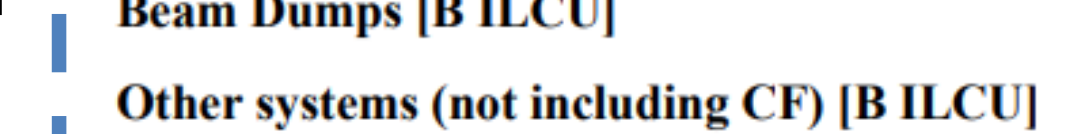 & & & $\begin{array}{l}0.21 \\
0.41\end{array}$ \\
\hline Tunnel length [km] & 20 & 20 & 20 \\
\hline Gradient $[\mathrm{MV} / \mathrm{m}]$ & & 31.5 & 31.5 \\
\hline$Q$ & $1 \times 10^{10}$ & $1 \times 10^{10}$ & $2 \times 10^{10}$ \\
\hline 1 Repectition rate $[\mathrm{Hz} \mid$ & & & 15 \\
\hline I Number of bunches & 1,312 & 2,624 & 2,624 \\
\hline | Beam power [MW] & & & 31.5 \\
\hline | Total R F pulse engith [ms & 1.618 & 2.35 & 2.35 \\
\hline
\end{tabular}

\section{Refurbishing a Cryomodule for High Gradient}

- In the context of ILC cost reduction, FNAL plans to lead the assembly of a High Gradient Cryomodule (HGC)

- Partners at national and international institutions such as JLab, Cornell, KEK, CEA, DESY, and TRIUMF

- CM1 will be disassembled and upgraded with cavities processed using new techniques developed since the ILC Technical Design Report

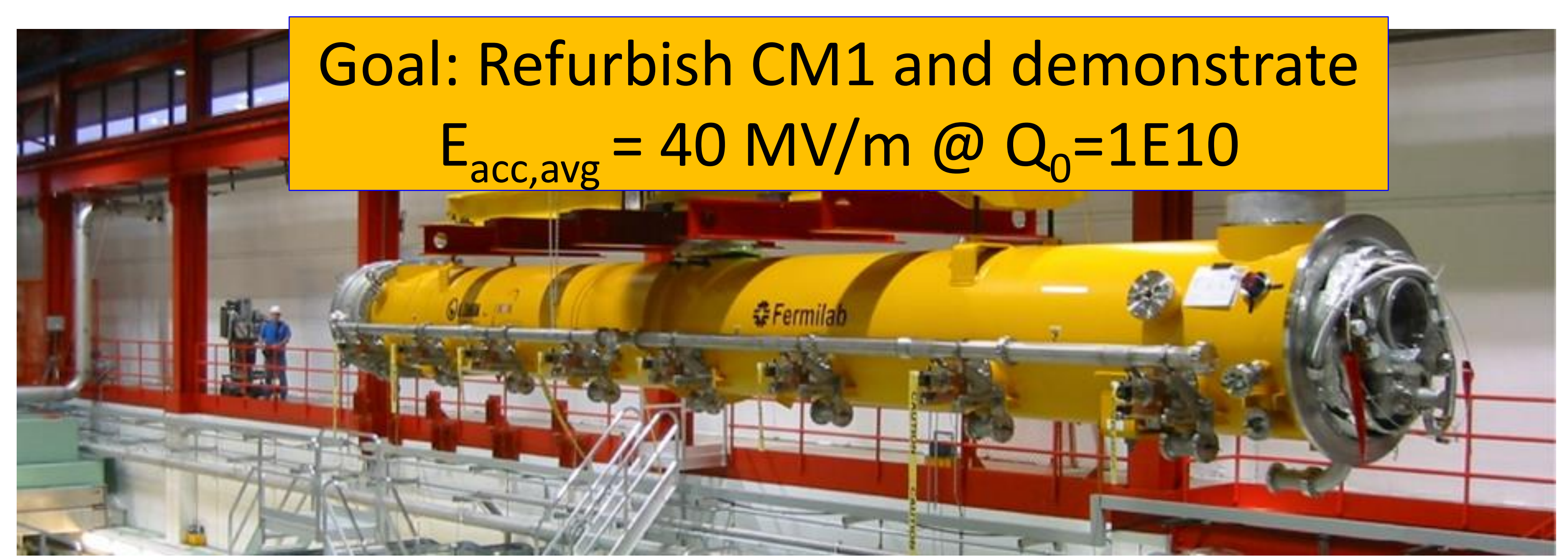

Unprecedented Performance in Single Cells with New High Gradient Surface Treatments

- Consistently achieve very high gradients with the FNAL developed $75 \mathrm{C} / 120 \mathrm{C}$ modified bake surface treatment
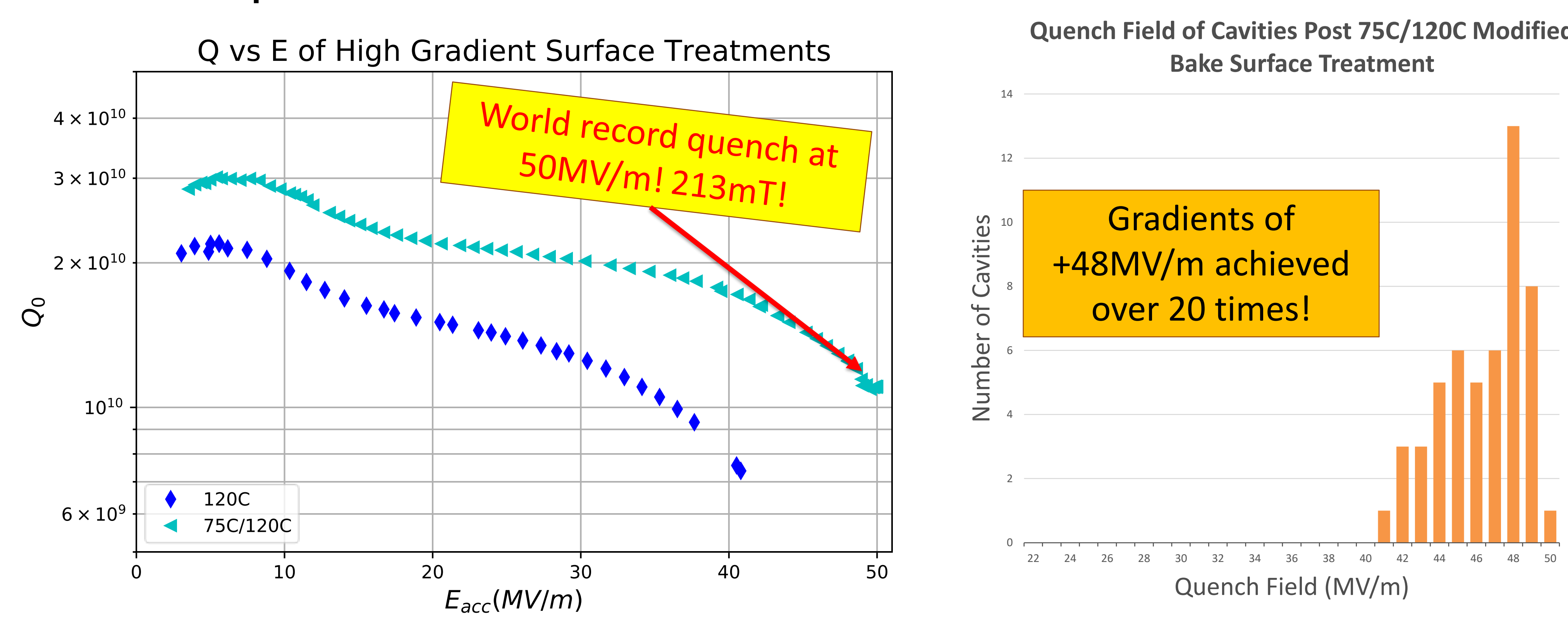

\section{Reproducibility of High Gradients at Other Labs}

- Two 1.3 GHz single cell cavities processed at FNAL with the $75 \mathrm{C} / 120 \mathrm{C}$ treatment were sent around the world for testing

- Very high gradients of +47 MV/m confirmed by other laboratories
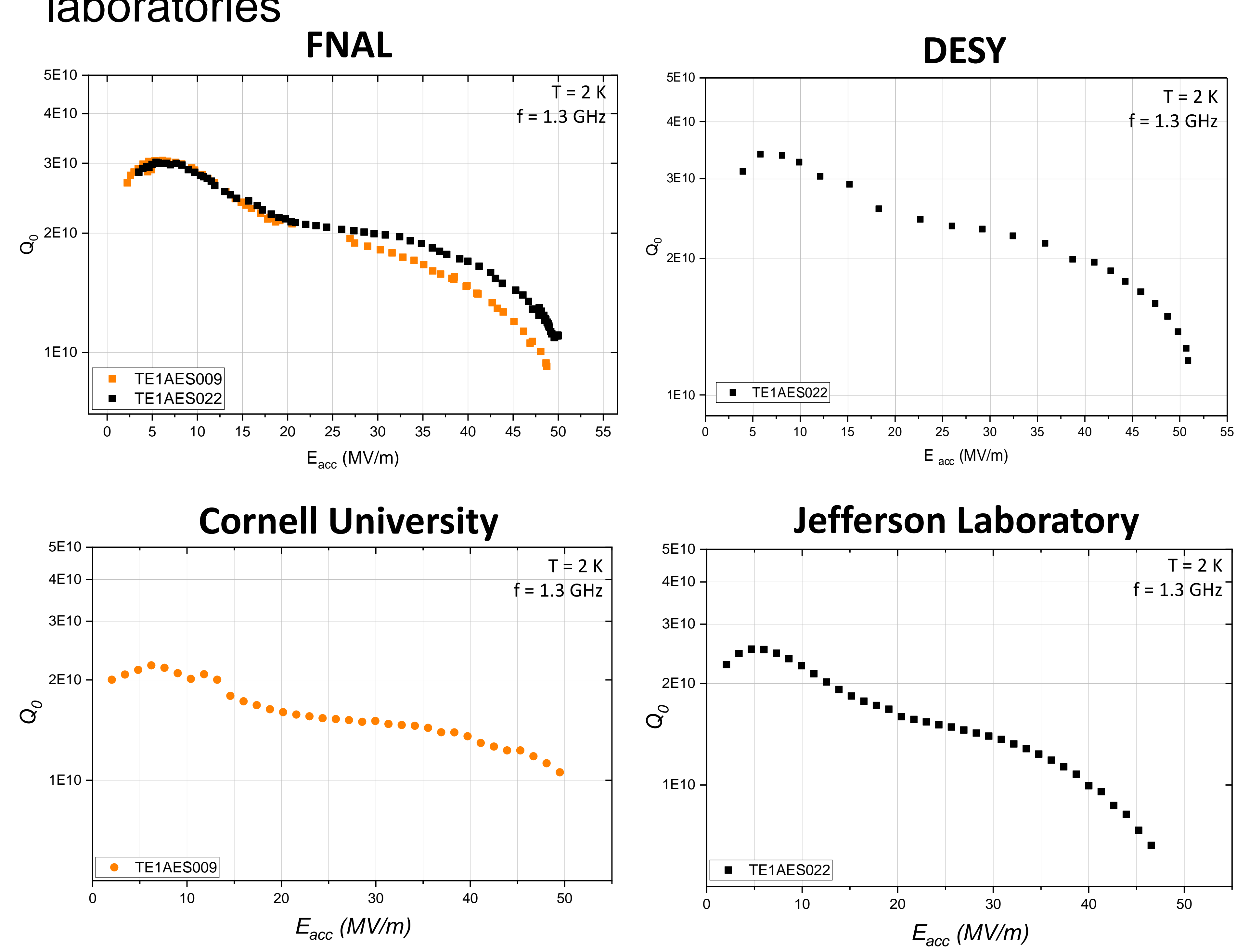

Material Science Studies to Uncover Mechanisms Responsible for Record High Gradients

- To better tailor surface treatments that further push the limits of record breaking performance, material science techniques are used to understand the microscopic mechanisms responsible for improved gradients and $Q_{0}$.

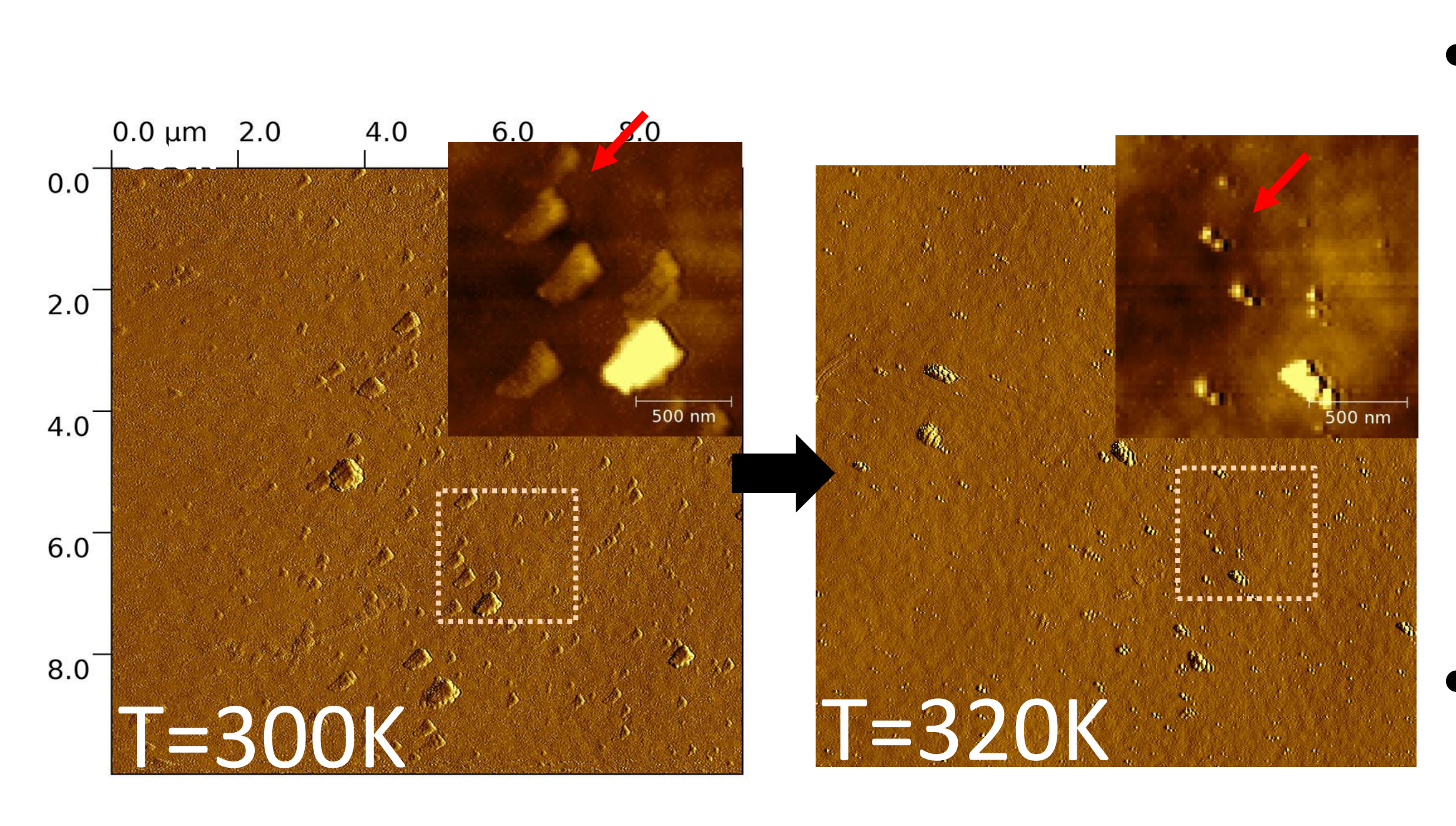

- Cryo Atomic Force Microscopy (AFM) images taken of $75 \mathrm{C} / 120 \mathrm{C}$ cavity cutouts show the growth and dissociation of nanohydrides

- Improved performance is linked to dissociation of these nano-hydrides

This manuscript has been authored by Fermi Research Alliance, LLC under Contract No. DE-AC02-07CH11359 with the U.S. Department of Energy, Office of Science, Office of High Energy Physics 